\title{
Comparison of Pre-Amputation Evaluation in Patients with and without Chronic Kidney Disease
}

\author{
Nivetha Subramanian ${ }^{a}$ Jialin Han ${ }^{b}$ Nicholas J. Leeper ${ }^{c}$ Elsie G. Ross ${ }^{c, d}$ \\ Maria E. Montez-Rath ${ }^{\mathrm{b}}$ Tara I. Chang ${ }^{\mathrm{b}}$ \\ aDepartment of Medicine, Stanford University School of Medicine, Stanford, CA, USA; ${ }^{b}$ Division of Nephrology, \\ Department of Medicine, Stanford University School of Medicine, Stanford, CA, USA; 'Division of Vascular Surgery, \\ Department of Surgery, Stanford University School of Medicine, Stanford, CA, USA; ${ }^{d}$ Center for Biomedical \\ Informatics Research, Stanford University School of Medicine, Stanford, CA, USA
}

\section{Keywords}

Peripheral artery disease $\cdot$ Amputation $\cdot$ Renal insufficiency

\begin{abstract}
Introduction: Patients with chronic kidney disease (CKD) and peripheral artery disease (PAD) are more likely to undergo lower extremity amputation than patients with preserved kidney function. We sought to determine whether patients with CKD were less likely to receive pre-amputation care in the 1-year prior to lower extremity amputation compared to patients without CKD. Methods: We conducted a retrospective observational study of patients with PAD-related lower extremity amputation between January 2014 and December 2017 using a large commercial insurance database. The primary exposure was CKD identified using billing codes and laboratory values. The primary outcomes were receipt of pre-amputation care, defined as diagnostic evaluation (ankle-brachial index, duplex ultrasound, and computed tomographic angiography), specialty care (vascular surgery, cardiology, orthopedic surgery, and podiatry), and lower extremity revascularization in the 1-year prior to amputation. We conducted separate logistic regression models to estimate the adjusted odds ratio (aOR) and $95 \%$ confidence intervals (Cls) among patients with and without
\end{abstract}

karger@karger.com www.karger.com/ajn

Karger"

GOPEN ACCESS
(C) 2021 The Author(s)

Published by S. Karger AG, Basel

This is an Open Access article licensed under the Creative Commons Attribution-NonCommercial-4.0 International License (CC BY-NC) (http://www.karger.com/Services/OpenAccessLicense), applicable to the online version of the article only. Usage and distribution for commercial purposes requires written permission.
CKD. We assessed for effect modification by age, sex, Black race, and diabetes status. Results: We identified 8,554 patients with PAD-related amputation. In fully adjusted models, patients with CKD were more likely to receive diagnostic evaluation (aOR 1.30; 95\% Cl 1.17-1.44) and specialty care (aOR $1.45,95 \% \mathrm{Cl} 1.27-1.64$ ) in the 1-year prior to amputation. There was no difference in odds of revascularization by CKD status (aOR 1.03, 0.90-1.19). Age, sex, Black race, and diabetes status did not modify these associations. Discussion/Conclusion: Patients with CKD had higher odds of receiving diagnostic testing and specialty care and similar odds of lower extremity revascularization in the 1-year prior to amputation than patients without CKD. Disparities in access to pre-amputation care do not appear to explain the higher amputation rates seen among patients with CKD.

(c) 2021 The Author(s)

Published by S. Karger AG, Basel

\section{Introduction}

Over 8 million people in the USA and over 200 million people worldwide have peripheral artery disease (PAD) $[1,2]$. PAD is strongly associated with significant morbidity and mortality, with an increased risk of developing cardiovascular disease and stroke [3, 4]. Chronic kidney 


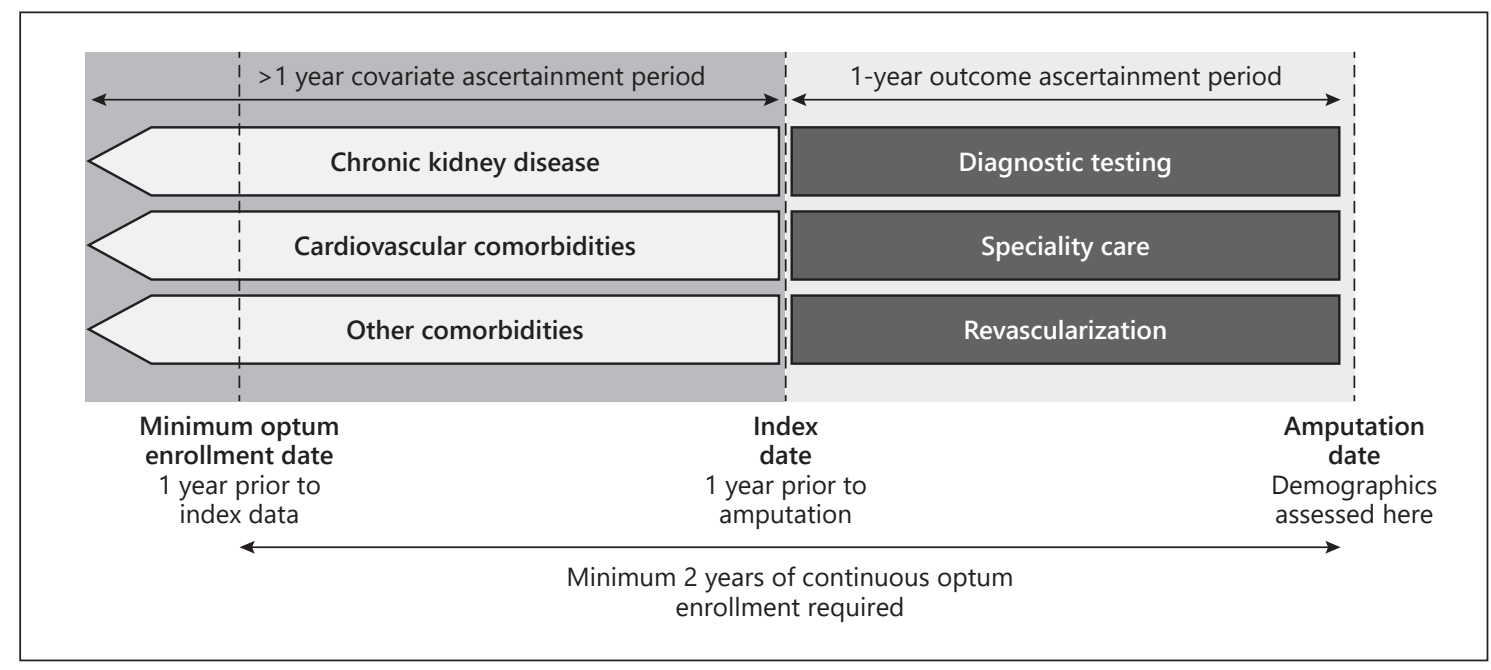

Fig. 1. Schematic diagram of the study design. All the patients underwent lower extremity amputation. Outcomes were assessed in the 1-year prior to amputation. Comorbid conditions, including CKD status, were ascertained starting from the index date, defined as 1-year prior to the amputation date. CKD, chronic kidney disease.

disease (CKD), which affects an estimated $14 \%$ of people in the USA [5], confers a markedly higher risk for PAD $[3,4,6,7]$.

Initially, PAD can be managed with lifestyle and risk factor modification to improve functional capacity and for secondary prevention of cardiovascular and cerebrovascular disease [8]. In individuals with claudication, defined as leg pain with exertion that improves with rest, cilostazol (a phosphodiesterase III inhibitor) and/or enrollment in an exercise training program have been shown to improve symptoms [8]. For those individuals who continue to experience disabling symptoms or develop rest ischemia (severe pain of the limb at rest), early revascularization therapy is critical to improve quality of life and possibly to prevent limb loss [8]. When medical or revascularization strategies have failed, lower extremity amputation is the final option but is associated with significantly higher mortality and morbidity, longer hospitalization stays, and negative effects on quality of life [4].

Patients with CKD and PAD are more likely to have lower extremity amputation than patients with PAD without CKD [9-13]. It is unclear whether this disparity is driven by differences in receipt of pre-amputation care, provider-level biases, treatment effectiveness, or other factors. We conducted the present analysis to test the hypothesis that patients with CKD would be less likely than patients without CKD to have diagnostic evaluation, specialty care visits, and lower extremity revascularization in the 1-year prior to lower extremity amputation.

\section{Methods}

Study Cohort

We used Optum's de-identified Clinformatics ${ }^{\circledR}$ Data Mart Database version 7.1 to conduct this analysis. Clinformatics ${ }^{\circledR}$ Data Mart Database is a de-identified database containing inpatient and outpatient medical claims, pharmaceutical claims, laboratory results, as well as socioeconomic and demographic information from commercial insurance providers and Medicare Advantage Plans [14]. This database comprises approximately $25 \%$ of all Medicare Advantage enrollees in the USA.

We included all adults aged 18 years and older who underwent a PAD-related lower extremity amputation from January 1, 2014, to December 31, 2017, identified using International Classification of Diseases, 9th and 10th Edition (ICD9 and ICD10) codes and Current Procedural Terminology (CPT-4) procedure codes (online suppl. Table 1; see www.karger.com/doi/10.1159/000516017 for all online suppl. material). We excluded patients with nonPAD causes of amputation, defined as having a ICD9 or ICD10 diagnosis code for trauma, congenital or acquired deformities, or malignancy during the same hospitalization as the lower extremity amputation [15]. For patients with multiple amputation events during this time period, we ascertained their first amputation event only. We defined the date 1-year prior to the amputation date as the index date (Fig. 1). We ascertained our exposure of interest (CKD status, detailed below) using all available data prior to the index date. Because we wanted all patients to have at least a 1-year ascertainment window for CKD status, we required that all patients have continuous enrollment in the Optum databases for at least 2 years prior to the amputation.

\section{Exposure: $C K D$}

Our primary exposure is CKD status, based on a qualifying ICD9 or ICD10 code or a qualifying laboratory value at any time 
prior to the index date (Fig. 1). We used the same ICD9 or ICD10 diagnosis codes for CKD used by the Centers for Disease Control and Prevention CKD Surveillance System program [16]. We did not exclude patients receiving maintenance dialysis or with a kidney transplant. For laboratory values, we ascertained all available outpatient serum creatinine values, urine albumin-to-creatinine ratio, and urine protein-to-creatinine ratio. We calculated the estimated glomerular filtration rate (eGFR) using the CKD Epidemiology Collaboration formula [17]. We considered an eGFR $<60$ $\mathrm{mL} / \mathrm{min} / 1.73 \mathrm{~m}^{2}$ and/or urine albumin-to-creatinine ratio $>30$ $\mathrm{mg} / \mathrm{g}$ and/or urine protein-to-creatinine ratio $>150 \mathrm{mg} / \mathrm{g}$ were categorized as evidence of CKD.

\section{Outcomes: Pre-Amputation Care}

We studied 3 outcomes in the 1-year post-index date: diagnostic imaging evaluation, specialty care, and surgical or endovascular LE revascularization (Fig. 1). We chose a 1-year observation window because previous studies showed that vascular procedures completed during this time are more likely to treat significant PAD [18]. Diagnostic evaluation was identified using CPT-4 codes for ankle-brachial index $(93,922,93,923$, and 93,924), lower extremity computed tomographic angiography $(75,635,73,706,72,191)$, magnetic resonance angiography $(73,725,72,198)$, duplex ultrasound $(93,925$ and 93,926$)$, or invasive angiography $(36,140$, $36,200,36,245,36,246,36,247,36,248$, and 76,350). We also used Healthcare Common Procedure Coding System codes to further ascertain the use of magnetic resonance angiography (C8912, C8913, C8914, C8918, and C8919). We defined specialty care as any encounter with vascular surgery, cardiology, podiatry, orthopedics, or wound care, identified using taxonomy codes. We ascertained lower extremity surgical and endovascular revascularization using ICD9, ICD10, and CPT-4 codes (online suppl. Table 2).

\section{Comorbidities}

Demographic variables including age, sex, education status, and geographic location were derived from Optum at the time of amputation. We defined comorbidities from ICD9 and ICD10 diagnosis codes using the Elixhauser Comorbidity Software [19]. These comorbidities include PAD, heart failure, valvular disease, coronary artery disease, cerebrovascular disease, hypertension, diabetes, pulmonary circulation disease, chronic pulmonary disease, obesity, hypothyroidism, solid tumor without metastasis, anemia (vitamin and iron deficiency), blood loss anemia, coagulation deficiency, liver disease, rheumatoid arthritis and collagen vascular disease, paralysis, other neurologic disorders, fluid and electrolyte disorders, weight loss, alcohol abuse, drug abuse, psychoses, and depression.

\section{Statistical Analysis}

We summarized binary variables as proportions and continuous variables as means. We compared patients with CKD to patients without CKD for each of the 3 outcomes in separate multivariable-adjusted logistic regression models, and report odds ratios (ORs) with 95\% confidence intervals (CIs). In our cohort, $22 \%$ of patients were missing at least one of the following variables: race, household income, region, gender, and education. To handle the missing data, we assumed the data to be missing at random and used multiple imputations by chained equations to impute 22 data sets $[20,21]$ stratified by CKD status. The imputation model included the 3 outcomes and all independent variables in the ad- justed models. A logistic regression model was fit separately to each of the imputed datasets and the resulting models were pooled using Rubin's rules. Adjusted models included all covariates in Table 1 plus interaction terms between age and certain comorbidities chosen through backward selection using a threshold of $p<0.05$. We assessed model fit at each step using Hosmer and Lemeshow goodness-of-fit test, with $p>0.05$ indicating good fit. We evaluated for effect modification of the association of CKD and the 3 specified outcomes by age (as restricted cubic spline with 5 percentile knots), female sex, Black race, and diabetes mellitus status and report stratified results for the dichotomous variables and $p$ values for interactions for all 4 of these variables. All analyses were performed using SAS Enterprise Guide, version 7.1 (SAS Institute, Inc., Cary, NC, USA).

\section{Results}

We identified 8,554 patients who underwent lower extremity amputation between January 2014 and December 2017 who met our inclusion and exclusion criteria (online suppl. Fig. 1). Among this cohort, 5,392 (63\%) had CKD: 5,186 (96.2\%) were identified based on ICD codes alone, 1,196 (22.2\%) from an ICD code and laboratory tests, and 206 (3.8\%) from laboratory tests alone. Very few patients with CKD had laboratory tests indicating significant albuminuria $(N=797)$ or proteinuria $(N=248)$. Patients with CKD were older, more often Black race, and had a higher prevalence of comorbid conditions including heart failure, hypertension, and diabetes (Table 1).

Overall, 4,629 (54.1\%) patients had a diagnostic evaluation of the lower extremity in the 1 -year prior to amputation, with most patients receiving an ankle-brachial index measurement or duplex ultrasound (Table 2). In unadjusted analysis, patients with CKD were more likely to have had diagnostic evaluation in the 1-year prior to lower extremity amputation than patients without CKD (unadjusted OR 1.63; 95\% CI 1.49-1.78), and this association remained significant after multivariable adjustment (adjusted OR 1.30; 95\% CI 1.17-1.44). The association did not vary by age (online suppl. Fig. 2a), sex, Black race, and diabetes mellitus status (Fig. 2a).

A total of $6,962(81.4 \%)$ patients had an encounter with a specialty care provider in the 1-year prior to amputation. Most patients were seen by cardiology or podiatry, and very few patients were seen by wound care (Table 2). Patients with CKD were more likely than patients without CKD to have seen a specialty care provider in the 1-year prior to lower extremity amputation (unadjusted OR 1.89; 95\% CI 1.70-2.11), and this association remained significant after multivariable adjustment (ad- 
Table 1. Baseline characteristics of the cohort study of persons who underwent lower extremity amputation between January 1, 2014, and December 31,2017, stratified by CKD status

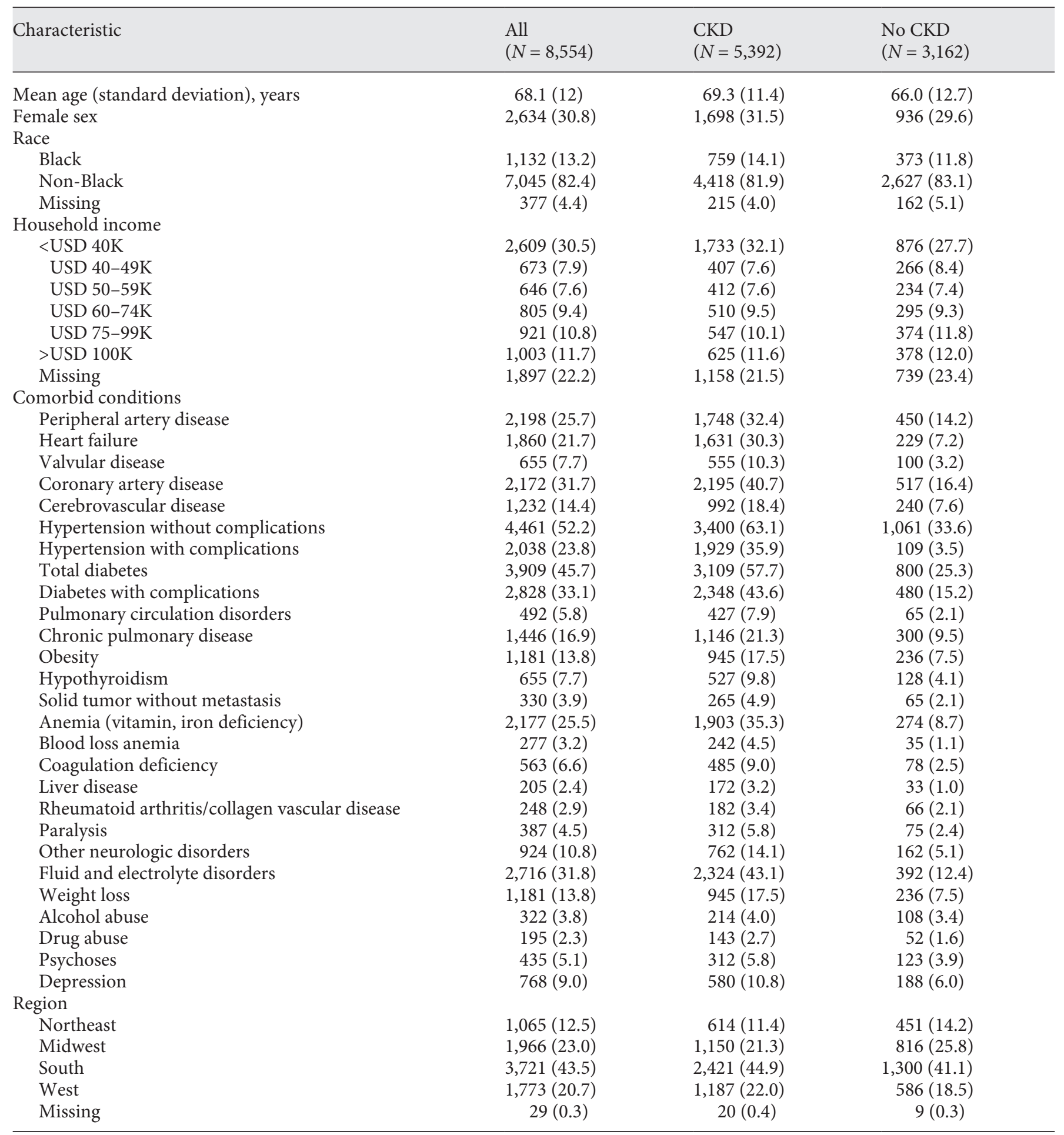

All values are $N(\%)$ except here indicated. CKD, chronic kidney disease.

Influence of Kidney Disease on Pre-Amputation Evaluation
Am J Nephrol 2021;52:388-395 DOI: $10.1159 / 000516017$ 
Table 2. Pre-amputation care received in the 1-year prior to amputation, stratified by CKD status

\begin{tabular}{lccc}
\hline Characteristic & $\begin{array}{l}\text { All } \\
(N=8,554)\end{array}$ & $\begin{array}{l}\text { CKD } \\
(N=5,392)\end{array}$ & $\begin{array}{l}\text { No CKD } \\
(N=3,162)\end{array}$ \\
\hline Diagnostic evaluation & & & \\
$\quad$ Ankle-brachial index & $3,237(37.8)$ & $2,191(40.6)$ & $1,046(33.1)$ \\
Computed tomographic angiography & $818(9.6)$ & $505(9.4)$ & $313(9.9)$ \\
Magnetic resonance angiography & $72(0.8)$ & $43(0.8)$ & $29(0.9)$ \\
Duplex ultrasound & $2,995(35.0)$ & $2,105(39.0)$ & $890(28.2)$ \\
Invasive angiography & $1,541(18.0)$ & $1,066(19.8)$ & $475(15.0)$ \\
Specialty care encounter & & & $761(24.1)$ \\
Vascular surgery & $2,515(29.4)$ & $1,754(32.5)$ & $1,415(44.8)$ \\
Cardiology & $4,720(55.2)$ & $3,305(61.3)$ & $1,509(47.7)$ \\
Podiatry & $4,611(53.9)$ & $3,102(57.5)$ & $556(17.6)$ \\
Orthopedics & $1,594(18.6)$ & $1,038(19.3)$ & $<10^{*}$ \\
Wound care & $<10^{*}$ & $<10^{*}$ & $270(8.5)$ \\
Revascularization & & & $342(10.8)$ \\
Surgical & $687(8.0)$ & $417(7.7)$ & $691(12.8)$ \\
$\quad$ Endovascular & $1,033(12.1)$ & & \\
\hline
\end{tabular}

All values are $N(\%)$. Patients may contribute to multiple categories within each outcome. * Unable to report due to cell size $N<10$. CKD, chronic kidney disease.

justed OR 1.45; 95\% CI 1.27-1.64). The association did not vary by age (online suppl. Fig. 2b), sex, Black race, or diabetes mellitus status (Fig. 2b).

Overall, 1,402 (16.4\%) of the cohort underwent lower extremity revascularization in the 1-year prior to amputation (Table 2). In unadjusted analysis, patients with $\mathrm{CKD}$ were more likely than patients without $\mathrm{CKD}$ to have undergone lower extremity revascularization in the 1 -year prior to lower extremity amputation (unadjusted OR 1.16; 95\% CI 1.03-1.31), but this association was no longer significant after multivariable adjustment (adjusted OR 1.03 ; 95\% CI 0.90-1.19). The association did not vary by age (online suppl. Fig. 2c), sex, Black race, and diabetes mellitus status (Fig. 2c).

\section{Discussion}

Our study found that in the 1-year prior to lower extremity amputation, compared with patients without $\mathrm{CKD}$, patients with CKD had higher odds of receiving diagnostic evaluations and specialty care, and similar odds of undergoing lower extremity revascularization. We found no evidence that age, sex, Black race, or diabetes status modified the association of CKD on pre-amputation care.
Our results were contrary to our hypothesis that patients with CKD would be less likely to have diagnostic testing and specialty care in the 1-year prior to lower extremity amputation. There are several potential explanations for our findings. First, patients with CKD could have had more severe PAD, which could lead to more extensive diagnostic testing and specialty care visits [22]. More severe neuropathy among patients with CKD could also lead to lower extremity wounds that require specialty care, although we did not see higher number of wound care encounters, which were uniformly low. Patients with CKD often present with atypical PAD symptoms, which may require more diagnostic imaging and coordination with specialty care to identify and treat [23]. Second, in our cohort, patients with CKD had a higher burden of concomitant cardiovascular disease, hypertension, and other comorbid conditions that could render them more likely to seek out specialty care or diagnostic testing for reasons beyond PAD management [23-25]. In fact, we did observe that over half of our cohort was seen by cardiology in the 1-year prior to amputation, which may have been for reasons unrelated to their limb. However, our results persisted after controlling for differences in comorbid conditions, demographic, socioeconomic, and geographic variables.

Our results also contrast with earlier studies indicating that patients with CKD were less likely than patients with more preserved kidney function to undergo revascular- 
Fig. 2. Forest plots showing the adjusted** OR and 95\% CIs for diagnostic evaluation (a); specialty care (b) and lower extremity revascularization for patients with $\mathrm{CKD}$ versus without $C K D$, overall and by specified subgroups $(\mathbf{c}) \cdot P_{\text {int }}=p$ values for interaction. ${ }^{* *} \mathbf{a}$ is adjusted for all covariates plus interaction between age (as a spline term) and pulmonary circulation disease, peripheral vascular disease, complicated hypertension, solid tumor without metastases, and alcohol abuse. ${ }^{* *} \mathbf{b}$ is adjusted for all covariates without additional interaction terms. ${ }^{* *} \mathbf{c}$ is adjusted for all covariates plus interaction between age (as a spline term) and sex, chronic pulmonary disease, iron deficiency anemia, and region. CKD, chronic kidney disease; CI, confidence interval; OR, odds ratio.

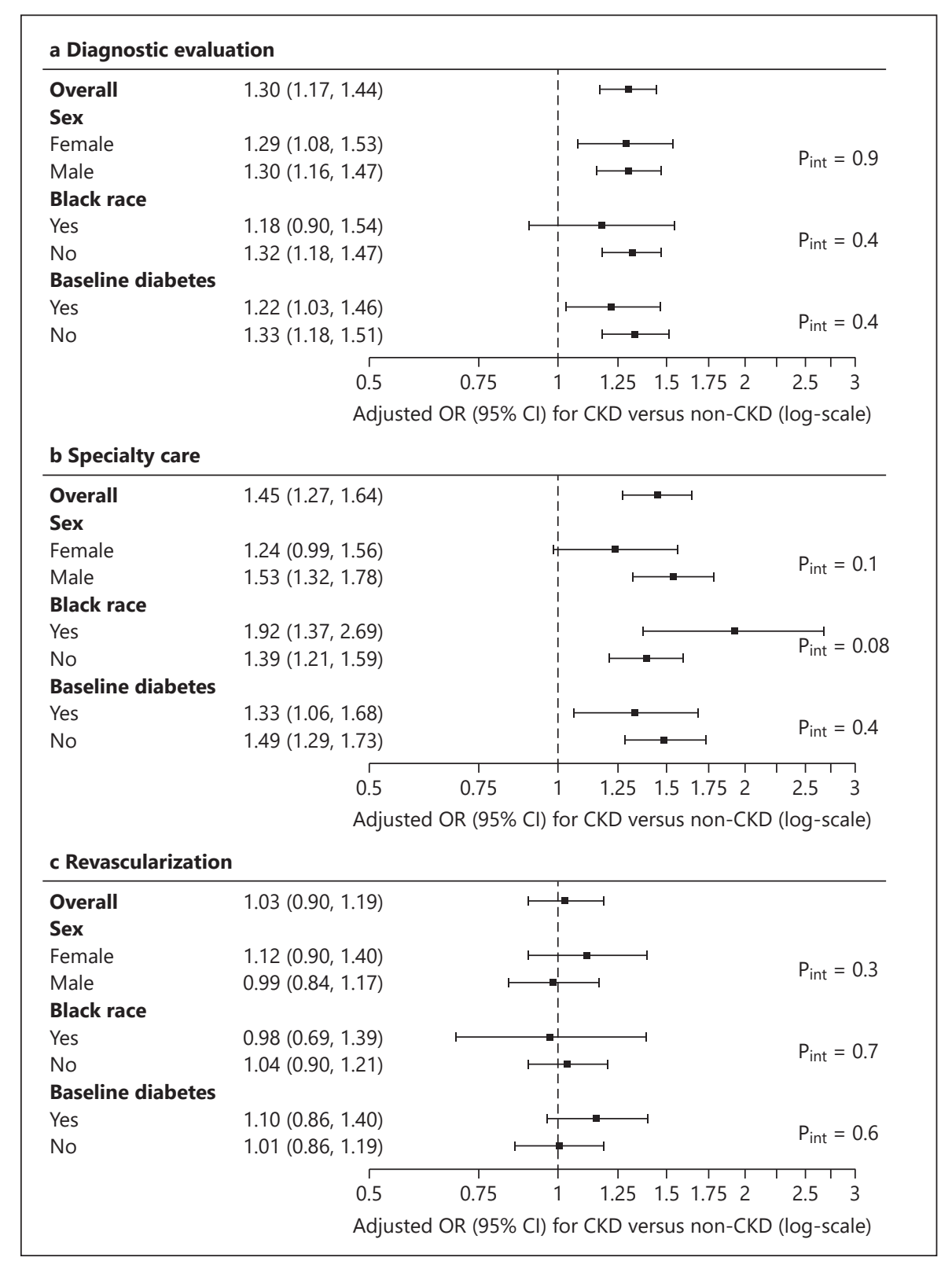

ization [26, 27]. Previous explanations for why patients with CKD were less likely to have revascularization included higher rates of complications, lower success rates, and concern for contrast-associated kidney injury in these patients [26, 27]. For example, in a study of male veterans presenting with critical limb ischemia, patients with an eGFR $30-59 \mathrm{~mL} / \mathrm{min} / 1.73 \mathrm{~m}^{2}$ had a $16 \%$ lower odds (CI 4-28\%) of undergoing lower extremity revascularization in the following 6 months compared to patients with an eGFR $\geq 60 \mathrm{~mL} / \mathrm{min} / 1.73 \mathrm{~m}^{2}$ [13]. That study was conducted from 2000 to 2002, while our study included patients from 2014 to 2017, and the differences in find- ings could reflect changes in practice patterns during the intervening years: increasing use of less invasive endovascular procedures and decreasing concerns regarding the clinical significance of contrast-associated kidney injury [28-30]. It is also possible that because all patients in our cohort ultimately went on to undergo lower extremity amputation, we selected for patients who were more likely to have had a revascularization procedure, regardless of CKD status.

Our study has several strengths, including the large, diverse cohort from many different regions in the USA. However, there are also some limitations. First, claims 
databases do not have information on lower extremity vascular anatomy such as length and location of the affected arteries [31], nor do they have information on the severity of PAD symptoms, which can affect choice of diagnostic testing or revascularization strategies. Second, most of our patients were categorized as having CKD or not based on claims information, which is highly specific but can lack sensitivity and could lead to miscategorization [32]. To mitigate these issues, we used a validated algorithm to ascertain CKD from claims and supplemented the information with laboratory values [16]. Third, since the Optum databases include only patients with private insurance, our results may not be generalizable to uninsured populations or patients with Medicare as their primary payer. Finally, despite adjustment for a large number of variables, residual confounding may have still affected our results.

In conclusion, using a large private insurance database, we found that patients with CKD had higher odds of receiving diagnostic testing and specialty care and similar odds of lower extremity revascularization in the 1 -year prior to amputation compared to patients without CKD. Taken together, our results suggest that while patients with CKD are interfacing more often with healthcare providers and undergoing more diagnostic imaging testing than patients without CKD, these encounters do not necessarily translate into more potentially limb-saving revascularizations. Whether this finding is due to the lesions being less amenable to revascularization in patients with CKD or to other patient-specific factors cannot be determined from our analysis. Further studies are needed to identify ways to deliver effective therapies that can reduce the risk of amputation in patients with CKD.

\section{Acknowledgements}

Data for this project were accessed using the Stanford Center for Population Health Sciences Data Core. The PHS Data Core is supported by a National Institutes of Health National Center for
Advancing Translational Science Clinical and Translational Science Award (UL1 TR001085) and from Internal Stanford funding. The content is solely the responsibility of the authors and does not necessarily represent the official views of the NIH.

\section{Statement of Ethics}

We used Optum's de-identified Clinformatics ${ }^{\circledR}$ Data Mart Database version 7.1 to conduct this analysis. Because the data are de-identified, this study was classified as exempt by the Stanford University Institutional Review Boards.

\section{Conflict of Interest Statement}

N.S.: none. J.H.: none. N.J.L. reports consulting fees from Janssen Pharmaceuticals unrelated to the current manuscript. E.G.R.: none. M.M.R.: none. T.I.C. has the following disclosures, which are not relevant to the current manuscript: funding paid by Janssen Pharmaceuticals to Stanford University for serving as a national leader for CREDENCE; consultancy for Bayer, Janssen Pharmaceuticals, Novo Nordisk, Fresenius Medical Care, Tricida, Gilead, and AstraZeneca; and received grant support from Satellite Healthcare.

\section{Funding Sources}

Support for this project was provided by a Stanford Cardiovascular Institute Seed Grant. The funder had no role in any aspect of the current manuscript.

\section{Authors Contributions}

Authors' contributions: research idea and study design: N.S., J.H., M.M.R., and T.I.C.; data acquisition: T.I.C.; data analysis/interpretation: all authors; statistical analysis: J.H. and M.M.R.; supervision or mentorship: N.J.L., E.G.R., M.M.R., and T.I.C. Each author contributed important intellectual content during the manuscript drafting or revision and accepts accountability for the overall work by ensuring that questions pertaining to the accuracy or integrity of any portion of the work are appropriately investigated and resolved.

\section{References}

1 Fowkes FG, Rudan D, Rudan I, Aboyans V, Denenberg JO, McDermott MM, et al. Comparison of global estimates of prevalence and risk factors for peripheral artery disease in 2000 and 2010: a systematic review and analysis. Lancet. 2013;382(9901):1329-40.

2 Hirsch AT, Duval S. The global pandemic of peripheral artery disease. Lancet. 2013; 382(9901):1312-4.
3 Chen SC, Chang JM, Liu WC, Tsai YC, Tsai JC, Hsu PC, et al. Brachial-ankle pulse wave velocity and rate of renal function decline and mortality in chronic kidney disease. Clin J Am Soc Nephrol. 2011;6(4):724-32.

4 Fowkes FG, Fowkes FG, Murray GD, Butcher I, Heald CL, Lee RJ, et al. Ankle brachial index combined with Framingham Risk Score to predict cardiovascular events and mortality: a meta-analysis. JAMA. 2008;300(2):197-208.
5 United States Renal Data System. USRDS annual data report: epidemiology of kidney disease in the United States. Bethesda, MD: National Institute of Diabetes and Digestive and Kidney Diseases, National Institutes of Health; 2018

6 Al-Qaisi M, Nott DM, King DH, Kaddoura S. Ankle brachial pressure index (ABPI): an update for practitioners. Vasc Health Risk Manag. 2009;5:833-41. 
7 Armstrong DW, Tobin C, Matangi MF. The accuracy of the physical examination for the detection of lower extremity peripheral arterial disease. Can J Cardiol. 2010;26(10):e346-50.

8 Swaminathan A, Vemulapalli S, Patel MR, Jones WS. Lower extremity amputation in peripheral artery disease: improving patient outcomes. Vasc Health Risk Manag. 2014;10: $417-24$.

9 Regensteiner JG, Hiatt WR, Coll JR, Criqui $\mathrm{MH}$, Treat-Jacobson D, McDermott MM, et al. The impact of peripheral arterial disease on health-related quality of life in the Peripheral Arterial Disease Awareness, Risk, and Treatment: New Resources for Survival (PARTNERS) Program. Vasc Med. 2008;13(1):1524.

10 Luders F, Furstenberg T, Engelbertz C, Gebauer K, Meyborg M, Malyar NM, et al. The impact of chronic kidney disease on hospitalized patients with peripheral arterial disease and critical limb ischemia. Angiology. 2017; 68(2):145-50.

11 Owens CD, Ho KJ, Kim S, Schanzer A, Lin J, Matros E, et al. Refinement of survival prediction in patients undergoing lower extremity bypass surgery: stratification by chronic kidney disease classification. J Vasc Surg. 2007; 45(5):944-52.

12 O'Hare AM, Bertenthal D, Shlipak MG, Sen S, Chren MM. Impact of renal insufficiency on mortality in advanced lower extremity peripheral arterial disease. J Am Soc Nephrol. 2005;16(2):514-9.

13 O'Hare AM, Bertenthal D, Sidawy AN, Shlipak MG, Sen S, Chren MM. Renal insufficiency and use of revascularization among a national cohort of men with advanced lower extremity peripheral arterial disease. Clin J Am Soc Nephrol. 2006;1(2):297-304.

14 Gunaseelan V, Kenney B, Lee JS, Hu HM. Databases for surgical health services research: clinformatics data mart. Surgery. 2019;165(4): 669-71.

15 Franz D, Zheng Y, Leeper NJ, Chandra V, Montez-Rath M, Chang TI. Trends in rates of lower extremity amputation among patients with end-stage renal disease who receive dialysis. JAMA Intern Med. 2018;178(8):102532.

16 Centers for Disease Control and Prevention. Prevalence of CKD in the Health Care System Population. 2020; Percentage of Patients with an ICD-9-CM or ICD-10-CM Code Indicating CKD. 2020. Available at: https://nccd.cdc. gov/ckd/Methods.aspx?Qnum=Q637. Accessed 2020 Aug 9.

17 Levey AS, Stevens LA, Schmid CH, Zhang YL, Castro AF, Feldman HI, et al. A new equation to estimate glomerular filtration rate. Ann Intern Med. 2009;150(9):604-12.

18 Goodney PP, Holman K, Henke PK, Travis LL, Dimick JB, Stukel TA, et al. Regional intensity of vascular care and lower extremity amputation rates. J Vasc Surg. 2013;57(6):1471-79, 1480.e1-3; discussion 1479-1480.

19 Agency for Healthcare Research and Quality. Elixhauser Comorbidity Software, Version 3.7. 2020. https://www.hcup-us.ahrq.gov/ toolssoftware/comorbidity/comorbidity.jsp. Accessed 2020 Aug 9.

20 Montez-Rath ME, Winkelmayer WC, Desai M. Addressing missing data in clinical studies of kidney diseases. Clin J Am Soc Nephrol. 2014;9(7):1328-35.

21 van Buuren S. Multiple imputation of discrete and continuous data by fully conditional specification. Stat Methods Med Res. 2007; 16(3):219-42.

22 Selvin E, Erlinger TP. Prevalence of and risk factors for peripheral arterial disease in the United States: results from the National Health and Nutrition Examination Survey, 1999-2000. Circulation. 2004;110:738-43.

23 Garimella PS, Hart PD, O'Hare A, DeLoach S, Herzog CA, Hirsch AT. Peripheral artery disease and CKD: a focus on peripheral artery disease as a critical component of CKD care. Am J Kidney Dis. 2012;60(4):641-54.

24 Luders F, Bunzemeier H, Engelbertz C, Malyar NM, Meyborg M, Roeder N, et al.
CKD and acute and long-term outcome of patients with peripheral artery disease and critical limb ischemia. Clin J Am Soc Nephrol. 2016;11(2):216-22.

25 Arinze NV, Gregory A, Francis JM, Farber A, Chitalia VC. Unique aspects of peripheral artery disease in patients with chronic kidney disease. Vasc Med. 2019; 24:25160.1358863X18824654.

26 Xie JX, Glorioso TJ, Dattilo PB, Aggarwal V, Ho PM, Barón AE, et al. Effect of chronic kidney disease on mortality in patients who underwent lower extremity peripheral vascular intervention. Am J Cardiol. 2017;119(4):66974.

27 Stavroulakis K, Borowski M, Torsello G, Bisdas T, Adili F, Balzer K, et al. One-year results of first-line treatment strategies in patients with critical limb ischemia (CRITISCH registry). J Endovasc Ther. 2018;25(3):320-9.

28 Goodney PP, Tarulli M, Faerber AE, Schanzer A, Zwolak RM. Fifteen-year trends in lower limb amputation, revascularization, and preventive measures among medicare patients. JAMA Surg. 2015;150(1):84-6.

29 Hiremath S, Velez JCQ. Preventing a nonexistent entity: the curious case of contrast and acute kidney injury. Curr Opin Nephrol Hypertens. 2020;29(1):152-60.

30 Davenport MS, Perazella MA, Yee J, Dillman JR, Fine D, McDonald RJ, et al. Use of intravenous iodinated contrast media in patients with kidney disease: consensus statements from the American College of Radiology and the National Kidney Foundation. Radiology. 2020;294(3):660-8.

31 Chen Q, Smith CY, Bailey KR, Wennberg PW, Kullo IJ. Disease location is associated with survival in patients with peripheral arterial disease. J Am Heart Assoc. 2013;2(5): e000304.

32 Grams ME, Plantinga LC, Hedgeman E, Saran R, Myers GL, Williams DE, et al. Validation of $\mathrm{CKD}$ and related conditions in existing data sets: a systematic review. Am J Kidney Dis. 2011;57(1):44-54.
Influence of Kidney Disease on

Pre-Amputation Evaluation
Am J Nephrol 2021;52:388-395

DOI: $10.1159 / 000516017$ 